\title{
Entanglement vs. Correlation in Quantum Theory
}

\author{
A. F. Kracklauer \\ Independent Researcher, Weimar, Germany \\ Email: af.kracklauer@web.de
}

How to cite this paper: Kracklauer, A.F. (2017) Entanglement vs. Correlation in Quantum Theory. Journal of Applied Mathematics and Physics, 5, 1035-1043. https://doi.org/10.4236/jamp.2017.55091

Received: March 1, 2017

Accepted: May 12, 2017

Published: May 16, 2017

Copyright $\odot 2017$ by author and Scientific Research Publishing Inc. This work is licensed under the Creative Commons Attribution International License (CC BY 4.0).

http://creativecommons.org/licenses/by/4.0/

c) (i) Open Access

\begin{abstract}
A detailed explication of Edwin Jaynes' criticism of Bell's deviation of his renowned inequality is presented. The exact consequence of an incorrect symbolic rendition for a conditional probability for the derivation is illustrated. Additionally, a data-point by data-point simulation of an optical test intended to exploit a Bell Inequality for demonstrating the inevitability of either irreality or nonlocality is described. This simulation shows in detail that only non correlated signal pairs do not violate the chosen form of a Bell Inequality, contrary to the intended hypothetical input into Bell's derivation. Finally, attention is drawn to the fact that, von Neumann's "Projection Hypothesis" is the basic conception behind nonlocality in quantum theory.
\end{abstract}

\section{Keywords}

Entanglement, Nonlocality, Irreality, Bell Inequality, Quantum Theory, Projection Hypothesis

\section{Introduction}

The discovery of Quantum Theory was achieved through fortuitous guesswork. In this regard, both Schroedinger and Heisenberg were successful. Schroedinger's version, however, has become dominant probably because it is based on a differential equation, as was (and is) the overwhelming rest of physics theory. The form of this equation is such that its solutions comprise a vector space (Hilbert space) with each basis vector being an eigenvector for which the totality of eigenvalues can be related to the observed spectrum of the electromagnetic emissions and adsorptions of the various elements. Thus, this equation must somehow reflect real, ontological structure of the material world. At the same time, however, the physical meaning of the eigenvectors or eigenfunctions themselves remains obscure. Ultimately they, according to Born, are to be interpreted as 
ontological entities, denoted "wave functions," by declaring that their modulus give the probability of presence of atomic sized entities (ultimately extended to all material entities regardless of size).

This interpretation, however, almost immediately led to a difficulty, namely, a wave function often had a larger spacial extent or other physical characteristics than those observed for its material correspondent. This problem was then resolved in theory by von Neumann who proposed the so-called "Projection Hypothesis" according to which the act of observation evokes a wave function "collapse" to the observed form. This process is not captured theoretically by Schroedinger's Equation; and, furthermore, it is unverifiable insofar as the actual state of a material entity before observation cannot be "observed" for empirical verification in its wave-function state. Nevertheless, nowadays it is a generally endorsed principle of Quantum Theory. See, e.g.: [1].

This is the state of quantum ontology most widely, but by no measure universally, promulgated as the conventional viewpoint.

The concept of "wave function collapse" caused by observation is not only weird but also ambiguous and anthropocentric. It seems to imply that material existence as it is observed requires something like human perception and involvement for realization. This feature has been denoted as the issue of "reality," as it seems to imply that the observed world is "realized" by the agency of human observation. In other words, the world as it is seen would not be as it appears were there no human observers to trigger wave collapse.

Although this matter alone is sufficient to be found disturbing, there is still another such feature of wave-function-ontology seeming implied by Quantum Theory. It is this: the wave function for a compound entity comprised of two or more sub entities, which after breaking apart such that the sub parts remain correlated, remains uncollapsed until any of the separated subparts are observed, when, so as to conserve various quantities, the wave function for all the parts collapses. This is to happen instantaneously even if the sub entities are separated by arbitrarily large distances, contrary to the Principle of "Einstein Causality" according to which no physical process (or "cause") can propagate faster than the speed of light. For historical reasons this effect is known nowadays in the terminology of field theory as "nonlocal" interaction.

In summary, measurement as described by Quantum Theory as generally presented nowadays admits the preternatural properties of "irreality" and nonlocality.

\section{Heterodoxical Investigations}

As to be expected from the start, some researchers openly sought alternative interpretations with the intention of exploring the possibility of precluding fantastical or preternatural features. Among many other conceptually distinct attempts, some researchers, including von Neumann, questioned whether quantum theory was an average or statistical shadow of a larger theory with additional variables such that, under averaging the hypothesized extra variable (s), it would project down onto the then (and now) existent formulation of Quantum Theory [2]. 
Von Neumann convinced himself that, in fact no such meta theory compatible with Quantum Theory could be found. Within a decade, however, it was seen that von Neumann's published proof of this conclusion contained a questionable premise [3].

Stimulated by the existence of deBroglie's Pilot Wave interpretation, John Bell, while understanding von Neumann's mistake, sought to reexamine the issue of the existence of a statistical meta theory by reformulating the hypothetical inputs into von Nemann's proof in physical rather than formalistic terms. See, e.g.: [4]. Specifically, Bell sought to realize this aim by setting as an hypothesis that there exists functional representations of correlated daughters of disassociated parent entities. Specifically, he considered a parent particle with zero spin composed of two daughters of opposite spin (equal to $\hbar / 2$ ) in the form of a function, $A$ say, for one daughter and $B$ for the other. Although Bell did not specifically declare such, at the time of his writing it was virtually universally accepted (mostly, however, implicitly) that, these symbolic expressions constituted wave functions, and must therefore represent the ontological essence of the daughter particles. Thus, it would have been natural for him to expect that these expressions are to be written in terms two sets of variables with values obtainable by some sort of measurement process of certain, but possibly unknown, variable(s) specifying properties pertaining exclusively to the daughter entities individually. For the first set, i.e., the "laboratory" variables, he employed the symbols $a,(b)$, and for the second set, pertaining to particle properties, $\lambda$; that is he wrote: $A(a, \lambda)$ and $B(b, \lambda)$. Thereupon, he assumed that, given their nature as symbolic representations of ontological entities, locality (i.e., Einstein locality or the ontic independence of any object on entities or conditions separated by a space-like interval) must also be symbolically manifest. This is effected, he asserted, by the independence of $A$ on $b$ as well as $B$ on $a$. Thus, for the wave function of the correlated daughters when separated, he wrote:

$$
P(a, b)=\int \mathrm{d} \lambda \rho(\lambda) A(a, \lambda) B(b, \lambda) .
$$

\section{Inequality Derivations}

Then, without further motivation, Bell used the following argumentation to deduce an inequality which appeared to be such that it could be tested empirically. The derivation proceeds as follows. Consider the difference of two such coincident probabilities:

$$
P(a, b)-P\left(a, b^{\prime}\right)=\int \mathrm{d} \lambda \rho(\lambda)\left[A(a, \lambda) B(b, \lambda)-A(a, \lambda) B\left(b^{\prime}, \lambda\right)\right] .
$$

Here, zero in the form:

$$
\begin{aligned}
0= & \int \mathrm{d} \lambda \rho(\lambda)\left[A(a, \lambda) B(b, \lambda) A\left(a^{\prime}, \lambda\right) B\left(b^{\prime}, \lambda\right)\right. \\
& \left.-A(a, \lambda) B\left(b^{\prime}, \lambda\right) A\left(a^{\prime}, \lambda\right) B(b, \lambda)\right],
\end{aligned}
$$

is added to Equation (2) to get:

$$
\begin{aligned}
P(a, b)-P\left(a, b^{\prime}\right)= & \int \mathrm{d} \lambda \rho(\lambda)\left[A(a, \lambda) B(b, \lambda)\left\{1+A\left(a^{\prime}, \lambda\right) B\left(b^{\prime}, \lambda\right)\right\}\right] \\
& -\int \mathrm{d} \lambda \rho(\lambda)\left[A(a, \lambda) B\left(b^{\prime}, \lambda\right)\left\{1+A\left(a^{\prime}, \lambda\right) B(b, \lambda)\right\}\right],
\end{aligned}
$$


which, using $|P| \leq 1$, can be written:

$$
\begin{aligned}
\left|P(a, b)-P\left(a, b^{\prime}\right)\right| \leq & \int \mathrm{d} \lambda \rho(\lambda)\left[\left\{1+A\left(a^{\prime}, \lambda\right) B\left(b^{\prime}, \lambda\right)\right\}\right] \\
& -\int \mathrm{d} \lambda \rho(\lambda)\left[\left\{1+A\left(a^{\prime}, \lambda\right) B(b, \lambda)\right\}\right] ;
\end{aligned}
$$

or

$$
\left|P(a, b)-P\left(a, b^{\prime}\right)\right| \leq 2+P\left(a^{\prime}, b^{\prime}\right)+P\left(a^{\prime}, b\right),
$$

i.e.,

$$
\left|P(a, b)-P\left(a, b^{\prime}\right)\right|+\left|P\left(a^{\prime}, b^{\prime}\right)+P\left(a^{\prime}, b\right)\right| \leq 2,
$$

which is one form of the celebrated "Bell inequalities." Numerous experiments have shown that data taken from various physical realizations of the input hypotheses (most often optical experiments on correlated polarization states ${ }^{1}$ ) have shown a clear violation of this inequality [6]. This is taken to mean that the hypothetical, local-realistic meta theory involving additional variables which, when averaged out, yields conventional Quantum Mechanics, does not exist. Further, this is taken to mean that the correlations between the quantum states has a extraordinary character for which nowadays the term "entangled" is used.

\section{Critique}

The argumentation as presented above is the current orthodoxy as presented in the majority of textbooks. Of course, there exist concurrently various critical, minority view points. It is the purpose of this note to present a logically complete rendition of one such view point, namely that apparently initiated by Edwin Jaynes in about 1987 [7]. Jaynes' central point was that Bell failed to correctly employ coincident probabilities by failing to distinguish the fact that they are the product of an absolute probability times a conditional probability. This had the consequence of introducing a logical error in Bell's reasoning.

Specifically, a coincidence probability, using Bell's notation, is correctly written as follows:

$$
P(a, b)=\int \mathrm{d} \lambda \rho(\lambda) A(a \mid b, \lambda) B(b \mid \lambda),
$$

where $B(b)$ is an absolute probability and $A(a \mid b)$ is a conditional probability. The presence of a variable pertaining to a remote detection process in $A$, i.e., $b$, does not imply, as Bell at least inadvertently took it, that there is a direct causative effect from the remote detector; rather only that, events at $A$ are to be included in a calculation of the coincidence probability only when there is also a detection event at $B$. In all applications of this concept (except in Bell's analysis) it is presumed that the responsible agent for the coincident, correlated events (when they occur) was a "prior cause," i.e., an event in the overlap of the past light cones of the measuring events at both $A$ and $B$. There is nothing

\footnotetext{
${ }^{1}$ As has been pointed out elsewhere [5], there is a serious conceptual error in the employ of polarization of electromagnetic waves for this purpose in that their intrinsic structure involves no quantum aspects.
} 
in experiments done to check a Bell inequality that prohibits prior causes; thus, there is also no logical reason to require instantaneous interaction (entanglement) in these experiments. In short, where Bell considered that he was encoding Einstein "locality," in fact he inadvertently encoded statistical independence or non correlation. Testing the inequality with correlated events, then, cannot yield coherent results.

Now, if Equation (8) is to give the average over the extra variables, i.e. the $\lambda$, at the meta level then the dependence on variables, $a,(b)$, pertaining to the environment including measuring apparatus, would be passively transferred to similar factors at the lower, statistical level of conventional quantum theory.

Thus, consider a repetition of the manipulations above starting, however, from the correct form in standard notation of Equation (1), namely:

$$
P(a, b)=\int \mathrm{d} \lambda \rho(\lambda) A(a \mid \lambda) B(b \mid a, \lambda) \cong A(a) B(b \mid a, \lambda) .
$$

Again, consider the difference of two correlation probabilities:

$$
P(a, b)-P\left(a, b^{\prime}\right)=\int \mathrm{d} \lambda \rho(\lambda)\left[A(a \mid \lambda, b) B(b, \lambda)-A(a \mid \lambda) B\left(b^{\prime} \mid a, \lambda\right)\right],
$$

and now the corresponding form for what should be an expression equaling zero:

$$
\begin{aligned}
0= & \int \mathrm{d} \lambda \rho(\lambda)\left[A(a \mid \lambda) B(b \mid a, \lambda) A\left(a^{\prime} \mid \lambda\right) B\left(b^{\prime} \mid a^{\prime}, \lambda\right)\right. \\
& \left.-A(a, \lambda) B\left(b^{\prime} \mid a^{\prime}, \lambda\right) A\left(a^{\prime} \mid \lambda\right) B(b \mid a, \lambda)\right],
\end{aligned}
$$

giving:

$$
\begin{aligned}
P(a, b)-P\left(a, b^{\prime}\right)= & \int \mathrm{d} \lambda \rho(\lambda)\left[A(a \mid \lambda) B(b \mid a, \lambda)\left\{1+A\left(a^{\prime} \mid \lambda\right) B\left(b^{\prime} \mid a^{\prime}, \lambda\right)\right\}\right] \\
& -\int \mathrm{d} \lambda \rho(\lambda)\left[A(a \mid \lambda) B\left(b^{\prime} \mid a, \lambda\right)\left\{1+A\left(a^{\prime}, \lambda\right) B(b \mid a, \lambda)\right\}\right] .
\end{aligned}
$$

Using, as above, $|P| \leq 1$, permits writing:

$$
\begin{aligned}
\left|P(a, b)-P\left(a, b^{\prime}\right)\right| \leq & \int \mathrm{d} \lambda \rho(\lambda)\left[\left\{1+A\left(a^{\prime} \mid \lambda\right) B\left(b^{\prime} \mid a^{\prime}, \lambda\right)\right\}\right] \\
& -\int \mathrm{d} \lambda \rho(\lambda)\left[\left\{1+A\left(a^{\prime} \mid \lambda\right) B(b \mid a, \lambda)\right\}\right] .
\end{aligned}
$$

So, here we arrive at the crux of the matter insofar as an equation in the form of Equation (7) is clearly seen not to follow in this case because the term $A\left(a^{\prime} \mid \lambda\right) B(b \mid a, \lambda)$ does not equal $P\left(a^{\prime}, b\right)$. In fact it is undefined, or nonsense, as it is the product of the absolute probability $A\left(a^{\prime} \mid \lambda\right)$ times the conditional probability $B(b \mid a, \lambda)$, which is not conditioned on $a^{\prime}$, but on $a$, thereby rendering the product of these two terms meaningless. The final, general conclusion is that a Bell inequality deduced with this mistake (i.e., overlooking this incompatibility) is invalid; likewise deductions from it are invalid.

\section{Analysis of the Symbolics}

A criticism of Bell analysis, such as that herein, is aimed at the current "quantum" theory analysis of the interrelationship (be it entanglement or correlation) as it is 
carried out to give the empirically verified, and therefore doxologically regarded rationalization. That is, the principle object of analysis is a sequence of common algebraic manipulations expressed with a specialized notation. The latter, for example, includes the "bra/ket" notation, i.e., $\langle x|| y$,$\rangle to denote a vector, an$ entity no different from that otherwise denoted with, for example, a bold face font. The involved vector (or covector) spaces, regardless of the notation, are the same. The algebraic manipulations required to obtain the conventional quantum expression for the correlation of polarization states, i.e., $\cos ^{2}(a-b) / 2$, as presented in text books on Quantum Mechanics, take very little symbol manipulation and call on nothing more erudite than elementary calculus involving trigonometric functions--with one exception. Thus, it follows that, if Quantum Theory incorporates some process, e.g.: entanglement, not found in pre quantum theory, then it must somehow encompass its essence in those mathematical manipulations yielding the empirically verified results. Should there be, then, an exact parallel between the presumed "quantum" and classical calculations, then there can be no aspect unique to just one or the other theory without at least specific and distinct definitions.

The exception mentioned above is the employ of what is called the "singlet" state: $\langle\psi|=\frac{1}{2}(\langle e|-\langle f|)$, where $\langle e|$ and $\langle f|$ are mutually exclusive states. The singlet state has many peculiar features, both formal and logical. While its use in certain calculations leads to empirically verified results, it, by authority of the "von Neumann Projection hypothesis," is, as written, never observed-tautologically so, insofar as any observation made on it should cause a collapse to one or the other of $\langle e|$ or $\langle f|$. Further, all properties of systems held to be represented by this state are in fact deduced from observations made on multiple copies of the physical system to which it pertains. All this together suggest that, the singlet state is a stand-in for an ensemble of entities while it itself is not in fact a member of the subject ensemble, but an artifact useful for certain calculations involving averages pertaining to the whole ensemble.

\section{Analysis by Simulation}

A very useful tactic for the study of the experiments done to test Bell Inequalities is to attempt to construct a faithful computer simulation of them in which alternate features can be added or substituted so as to explore their consequences on the output data. While there may be considerable dispute over how to simulate various theoretical notions as expressed in the mathematical algorithms concerning the phenomena under study, there is much less room for dispute over how to model the experimental setup. For present purposes, the original experimental arrangement as proposed by Bell in the original paper in which he presented his analysis leading to his renowned inequality is central. Here it should be possible to model two scenarios: one in which the signals sent to measuring stations $A$ and $B$ are correlated as he specified, and another in which they are uncorrelated, as is noted herein is implicit in the form Bell inadvertently used in his inequality derivation. If such a comparison shows that correlated 
signals violate the inequality, but non correlated signals do not, then the criticism raised first by Jaynes is demonstrated empirically.

Fortunately, for simulating these experiments, the physical action on signals by the relatively few required optical devices are well known, and in this case are all based on application of Malus' Law. Thus, a simulation routine for Bell's initiatory experiment essentially consists of two blocks of code, the first generates the signals selectively as correlated or uncorrelated, while the second block applies Malus' Law to determine the probability of a detection. Also, to model the effect of inefficient detectors, an additional probabilistic pass/fail test can be applied to the results from that code counting detections. See Figure 1.

Results confirm that uncorrelated input signals result in the Bell inequality being satisfied, while correlated input signals violate the inequality in very close numerical approximation to the degree observed in data produced by optical laboratory experiments.

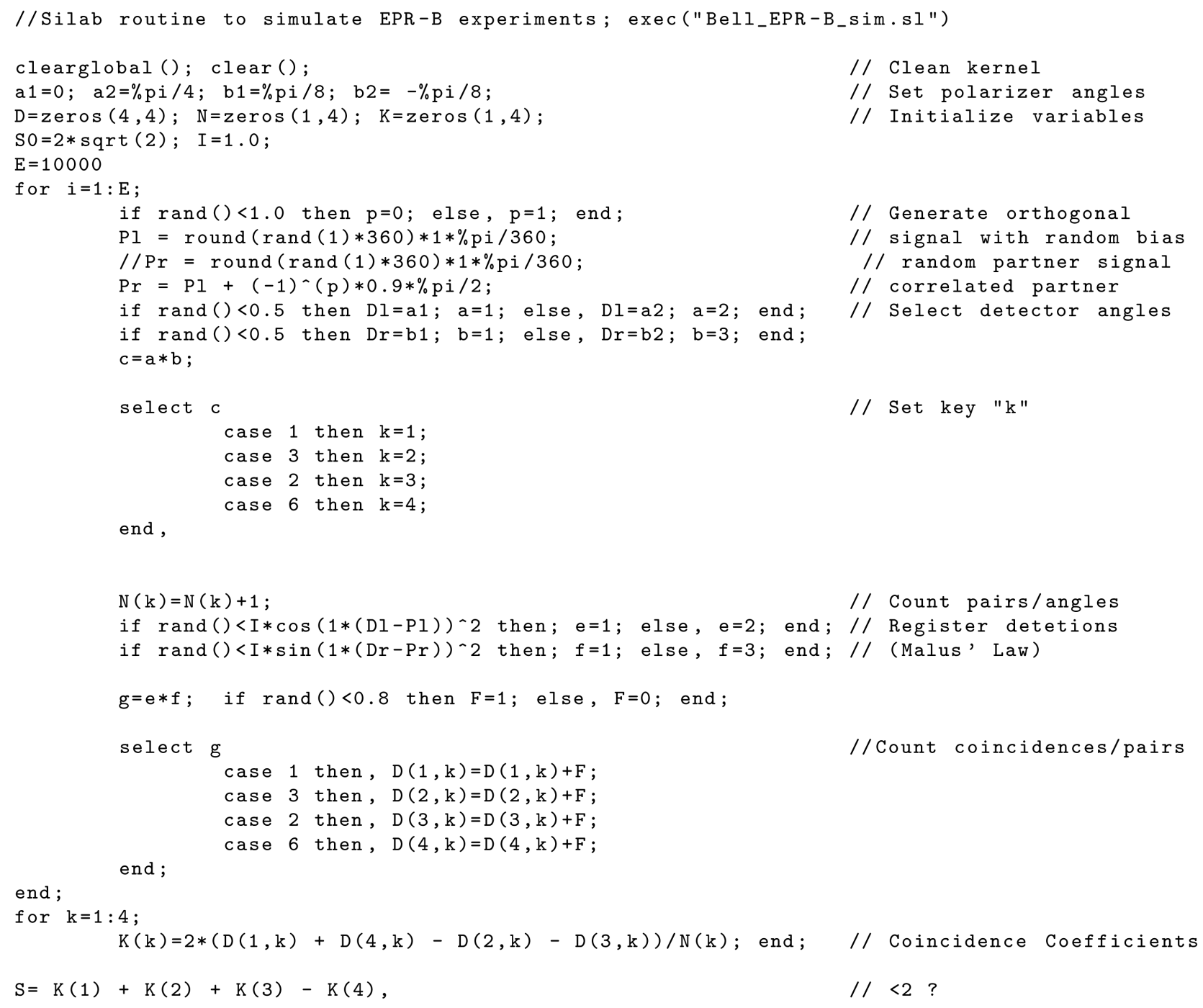

Figure 1. A Scilab routine to simulate point-wise an optical Bell test experiment. The type of input signals, correlated or not, is to be selected in the first block. The detector efficiency is set by specifying $I$. 


\section{Conclusions}

In conclusion it is natural to ask: what has been proven here, and what not? Surprisingly little actually, by cause of a virtually universally overlooked technicality. It is this: the space in which these experiments have been and are being done is devoid of quantum structure from the start. In technical terms, the space spanned by the two states of polarization, namely $S U(2)$, is in fact homeomorphic to $S O(3)$. The latter group is that for angular displacements on the surface of a sphere in three dimensions. It is well known and easily seen that the noncommutivity of displacements in longitude and latitude on a 3-D sphere results from topology and geometry and not from the dynamical structure of quantum theory, which is intimately related to the uncertainty principle. The homeomorphism of the relevant spaces implies that noncommutivity in each must have the same cause: geometry. Indeed, if a vector in $S O(3)$ is chosen, it will have two accompanying orthogonal vectors. As the vector in $S O(3)$ then is displaced, its orthogonal partners will be displaced along with it as a rigid triad. The group of motions for the two dimensional orthogonal pair is $S U(2)$, otherwise known as "Q-space," "spin space" or "polarization space." The structure within this space is not quantum mechanical. Quantum structure is restricted to two spaces: phase space, $\{q, p\}$ and "squeeze space," $\{\phi, A\}$ i.e., phase and amplitude.

Given these mathematical realities, it is indisputable that experiments on entities described by $S U(2)$ cannot resolve issues peculiar to Quantum Theory. On this basis, Bell's analysis and experimentation consequent to it, do not support the contention that "quantum entanglement" is a valid concept. If such a notion is in fact extant and useful in Quantum Mechanics, then it must be revealed in connection with phenomena described in quantized phase or squeeze space.

Interestingly, the space considered by Einstein, Podolsky and Rosen in their seminal article on this topic was phase space [8]. In other words, a space in which, if the phenomena related to "entanglement" nowadays in fact existed as quantum peculiarities, they could have been found in principle. Nevertheless, on the basis of the arguments presented herein, the notions of 1) ontological (rather than pure informational) wave functions, 2) wave collapse by cause of measurement and instantaneous collapse across arbitrarily large space-like separations can all be criticized as empirically unverified. And, the results of many Bell-test optical experiments can all be explained as manifestations of ordinary "priorcause" correlation.

\section{References}

[1] Friebe, C., Kuhlmann, M., Lyre, H., Naeger, P., Passon, O. and Stoeckler, M. (2015) Philosophie der Quantenphysik. Springer, Berlin, Heidelberg.

[2] von Neumann, J. (1932) Mathematische Grundlagen der Quantenmechanik. Springer, Berlin, 109

[3] Hermann, G. (1935) Die Naturphilosophischen Grundlagen der Quantenmechanik. Abhandlungen de Fries'schen Schule, 4, 75. 
[4] Bell, J.S. (1987) Speakable and Unspeakable in Quantum Mechanics. Cambridge University Press, Cambridge.

[5] Kracklauer, A.F. (2015) Entanglement: A Contrarian View. Journal of Modern Physics, 6, 1961-1968. https://doi.org/10.4236/jmp.2015.613202

[6] Bouwmeester, D., Ekert, A. and Zeilinger, A., Eds. (2000) The Physics of Quantum Information. Springer, Berlin, Heidelberg.

[7] Jaynes, E.T. (1989) Clearing up Mysteries-The Original Goal. In: Skilling, J., Ed., Maximum-Entropy and Bayesian Methods? Kluwer, Dordrecht, 1.

[8] Einstein, A., Podolsky, B. and Rosen, N. (1935) Can the Quantum-Mechanical Description of Physical Reality Be Considered Complete? Physical Review, 47, 777. https://doi.org/10.1103/physrev.47.777

Submit or recommend next manuscript to SCIRP and we will provide best service for you:

Accepting pre-submission inquiries through Email, Facebook, LinkedIn, Twitter, etc. A wide selection of journals (inclusive of 9 subjects, more than 200 journals)

Providing 24-hour high-quality service

User-friendly online submission system

Fair and swift peer-review system

Efficient typesetting and proofreading procedure

Display of the result of downloads and visits, as well as the number of cited articles

Maximum dissemination of your research work

Submit your manuscript at: http://papersubmission.scirp.org/

Or contact jamp@scirp.org 